\title{
A METHOD FOR COMPARING UNIVALENT FUNCTIONS
}

\author{
BY GERALD S. GOODMAN
}

Communicated by Dana Scott, September 6, 1968

1. The Loewner representation. Let $f$ be any function in the class $S$ of univalent functions on the unit disk bearing the normalization $f(0)=0, f^{\prime}(0)=1$. Then it is known [1], [2], that $f$ can be represented as

$$
f(z)=\lim _{z \rightarrow \infty} e^{t} h(z, t),
$$

locally uniformly in $z$ on $|z|<1$, where $h(z, \cdot)$ is the solution of Loewner's equation in its general form

$$
\frac{d h}{d t}=-h p(h, t) \quad \text { a.e. in } t \text { on }[0, \infty)
$$

with the initial values

$$
h(z, 0)=z, \quad|z|<1 .
$$

Here $p(\cdot, t)$ denotes a suitably chosen one-parameter family of holomorphic functions on the unit disk having positive real part and normalized so that $p(0, t) \equiv 1$, whose dependence on $t$ is Lebesgue measurable on $[0, \infty)$ whenever the first variable is held fixed, and the solution of (2) is understood in the Caratheodory sense [3].

Conversely, if $p(\cdot, t)$ denotes any family of functions satisfying the above requirements, then the solution to the foregoing initialvalue problem is known to exist and be holomorphic and univalent in $z$ on the unit disk; the limit in (1) is then also known to exist and to determine a function in class $S[1],[2]$.

More generally, one can consider the general solution $h(z, s, t)$ of (2) for $0 \leqq s \leqq t$ with the initial values

$$
h(z, s, s)=z, \quad|z|<1 .
$$

Then, in place of (1), one has

$$
\lim _{z \rightarrow \infty} e^{t} h(z, s, t)=g(z, s)
$$

locally uniformly in $z$ for $|z|<1$, where $e^{-\triangleleft} g(z, s)$ now belongs to $S$ for all $s$ in $[0, \infty)$. The function $g$ is absolutely continuous in $s$ and constitutes an integral of Equation (2), for it is easily shown that 


$$
g(h(z, t), t)=f(z)
$$

for all $t$ in $[0, \infty),[1],[2]$, and, in particular, that

$$
g(z, 0)=f(z) \text {. }
$$

2. Comparisons, local and global, in the class $S$. The identity (4) can be used as the basis for a local variational theory within the class $S$, by subjecting the unit disk in the $h$-plane to the infinitesimal transformations of the semigroup of bounded univalent functions, which Loewner has characterized [5]. This results in a variation in the function $f$ for each fixed $t$, and, in e.g., the case of extremal problems for coefficients of functions in class $S$, it leads to a condition on the initial coefficients of the functions $p(\cdot, t)$ that generate extremal mappings that amounts to the Pontryagin maximum principle [4], and is equivalent to Schiffer's characterization of the extremal functions as solutions of his quadratic differential equation [1], [6].

What we wish to report on now is the possibility of making global comparisons between the functions in class $S$, in contrast to the local comparisons mentioned above, by considering in place of (4) the function $G(z, t)$ defined by the equation

$$
g(\hat{h}(z, t), t)=G(z, t) \quad(|z|<1, \quad 0 \leqq t<\infty) .
$$

Here $h$ is understood to be the solution to the initial-value problem made up of (2) and (3) when $p(\cdot, t)$ is replaced by any other oneparameter family $\hat{p}(\cdot, t)$ that satisfies the same conditions as $p(\cdot, t)$.

The function $G$ belongs to class $S$ for all $t$ in $[0, \infty)$ and satisfies

$$
G(z, 0)=f(z)
$$

while (by arguments similar to those already used in [1], [2])

$$
\lim _{z \rightarrow \infty} G(z, t)=\hat{f}(z),
$$

locally uniformly in $z$ on the unit disk, where $\hat{f}$ is the function in $S$ generated by $\hat{h}$ in the same manner as (1). Moreover, $G$ is absolutely continuous in $t$, locally uniformly in $z$, and its $t$-derivative is given by

$$
\partial_{\imath} G(z, t)=\ddot{h}(z, t) g^{\prime}(h(z, t), t)[p(\ddot{h}(z, t), t)-\not h(h(z, t), t)] \quad \text { a.e., }
$$

where the ' denotes the derivative of $g$ w.r.t. its first argument.

This procedure makes it possible to join a given function $f$ in $S$ with any other function $\hat{f}$ in $S$ along an absolutely continuous path in $S$, thereby generalizing the procedure of $\$ 1$, which constitutes the special case of $f(z)=z$ in the present set-up. 
3. Application to extremal problems for coefficients of functions in $S$. If we expand both sides of (8) in power series about the origin, we find that the $n$th coefficient of $\partial_{t} G$ is given by the expression

$$
\sum_{m=1}^{n-1} B_{m}(t)\left[p_{m}(t)-p_{m}(t)\right] \quad \text { a.e., }
$$

where the $p_{m}$ and $p_{m}$ are the $m$ th coefficients of $p(\cdot, t)$ and $\hat{p}(\cdot, t)$, resp., and the $B_{m}$ are certain combinations of the initial coefficients of the functions $g$ and $h$ making them absolutely continuous as functions of $t$. Their derivatives involve the $p_{m}$ and $\hat{p}_{m}$, and when $p(\cdot, t)$ coincides with $\hat{p}(\cdot, t)$ the resulting expressions reduce to a set of differential equations which are already known in the local theory [1], [6].

To establish the global extremality in $S$ of the real part of the $n$th coefficient of a function $f$ generated by (2), it would clearly be enough to show that the real part of (9) is nonpositive for a.e. $t$ whatever the choice of the $\hat{p}_{m}$ (so long as they come from functions admissible in the sense of $\$ 1$ ), for then the real part of the $n$th coefficient of the function $G$ would be nonincreasing. In view of (6) and (7), the global extremal property would thereby be verified.

In practice, it is desirable to restrict the competing functions $\hat{p}(\cdot, t)$ by placing a limitation on the range of their initial coefficients. In certain cases it can be shown by using the symmetries of the coefficient body $S_{n}$ for functions in $S$ that this limitation results in no loss of generality.

4. Illustration: the case $n=3$. We shall verify the extremal property of the third coefficient of the Koebe function $f(z)=z /(1-z)^{2}$ in the class of functions $\hat{f}(z)=z+\hat{a}_{2} z^{2}+\hat{a}_{3} z^{3}+\cdots$ in $S$ which have $\operatorname{Re} \hat{a}_{2} \geqq 0$; an analogous result will hold for $f(z)=z /(1+z)^{2}$ when Re $\hat{a}_{2} \leqq 0$. We have $p(h, t)=(1-h) /(1+h)$ and $g(h, t)=e^{t} h /(1-h)^{2}$; if we put $\hat{p}(h, t)=1+2 \sum_{m=1}^{\infty} \hat{p}_{m}(t) h^{m}$ and $\hat{h}(z, t)=e^{-t}\left(z+\sum_{m=2}^{\infty} \hat{b}_{m}(t) z^{m}\right)$ then the real part of (9) for $n=3$ becomes twice

$$
\operatorname{Re}\left\{B_{1}(t)\left[-1-\hat{p}_{1}(t)\right]+B_{2}(t)\left[1-\hat{p}_{2}(t)\right]\right\},
$$

where

$$
B_{1}(t)=2 \hat{b}_{2}(t) e^{-t}+4 e^{-2 t}, \quad B_{2}(t)=e^{-2 t} .
$$

We compute from (2)

$$
\begin{aligned}
\frac{d \hat{b}_{2}(t)}{d t} & =-2 p_{1}(t) e^{-t} \text { a.e., } \\
\operatorname{Re} \frac{d \hat{b}_{2}(t)}{d t} & =\operatorname{Re}\left\{-4 \hat{b}_{2}(t) p_{1}(t) e^{-t}-2 p_{2}(t) e^{-2 t}\right\} \text { a.e., }
\end{aligned}
$$


and therefore

$$
\frac{d B_{1}(t)}{d t}=-B_{1}(t)+4 e^{-2 t}\left[-1-p_{1}(t)\right] \quad \text { a.e. }
$$

while from (11) we deduce that $B_{1}(0)=4$.

In order to make use of the assumption that $\operatorname{Re} \hat{a}_{2} \geqq 0$, we prove the following lemma.

LEMMA. If $\hat{a}_{2}=\lim _{t \rightarrow \infty} \hat{b}_{2}(t)$ has nonnegative real part, then the point $\left(\hat{a}_{2}, \operatorname{Re} \hat{a}_{3}\right)$ can be reached by a solution of (12) that starts at the origin when $t=0$ and has $\operatorname{Re} \hat{p}_{1}(t) \leqq 0$ a.e. in $[0, \infty)$.

PROOF. In view of (12), this is the same as saying that $\operatorname{Re} \hat{b}_{2}(t)$ can be assumed to be monotone nondecreasing. Suppose it is not. Then there will be two values $\xi<\eta$ of $t$ such that $\operatorname{Re} \hat{b}_{2}(\xi)=\operatorname{Re} \hat{b}_{2}(\eta)$. On the interval $[\xi, \eta]$ we can, if need be, replace $\hat{p}(\cdot, t)$ by the functions (also of positive real part)

$$
\begin{aligned}
q(h, t)=\frac{1}{2}\left[\hat{p}(h, t)+(\hat{p}(-\bar{h}, t))^{-}\right] & \\
& =1+2 i \operatorname{Im} p_{1}(t) h+2 \operatorname{Re} p_{2}(t) h^{2}+\cdots .
\end{aligned}
$$

This makes $\operatorname{Re} \hat{b}_{2}(t)$ constant on $[\xi, \eta]$ and leaves $\operatorname{Im} \hat{b}_{2}(t)$ unchanged, while in the equation for $\operatorname{Re} d \hat{b}_{3}(t) / d t$ the only change is that the term

$$
-4 \operatorname{Re} \hat{b}_{2}(t) \operatorname{Re} \hat{p}_{1}(t) e^{-t}
$$

is now missing. But

$$
\int_{\xi}^{\eta}-4 \operatorname{Re} \hat{b}_{2}(t) \operatorname{Re} \hat{p}_{1}(t) e^{-t} d t=\left[\operatorname{Re} \hat{b}_{2}(\eta)\right]^{2}-\left[\operatorname{Re} \hat{b}_{2}(\xi)\right]^{2}=0,
$$

so that the missing term does not affect the value of $\operatorname{Re} \hat{b}_{3}(\eta)$. By the Rising Sun Lemma, there are at most a countable number of disjoint intervals in $[0, \infty)$ where this alteration of $\hat{p}(\cdot, t)$ needs to be made, so that the altered $\hat{p}(\cdot, t)$ remains measurable in $t$ and yields a trajectory of (12) that satisfies the assertion of the lemma.

A similar reasoning, in which $\hat{p}(h, t)$ is replaced by

$$
q(h, t)=\frac{1}{2}[\hat{p}(h, t)+(\not(h, t))-],
$$

shows that we can also assume that $\operatorname{Im} \hat{p}_{1}(t)$ does not change sign on $[0, \infty)$. For $\operatorname{Re} p_{1}(t)$ and $\operatorname{Im} p_{1}(t)$ restricted in this way, Equation (13) and the initial condition $B_{1}(0)=4$ imply that

$$
\operatorname{Im} B_{1}(t) \operatorname{Im} \hat{p}_{1}(t) \leqq 0 \text { a.e., }
$$


and

$$
4 e^{-2 t} \leqq \operatorname{Re} B_{1}(t) \leqq 4 e^{-t}
$$

(since now $0 \leqq 1+\operatorname{Re} p_{1}(t) \leqq 1$ a.e.).

To prove that (10) is nonpositive it is therefore enough to show that

$$
-4\left[1+\operatorname{Re} p_{1}(t)\right] \leqq \operatorname{Re} p_{2}(t)-1 \text { a.e. }
$$

We may restrict $p_{1}(t)$ and $p_{2}(t)$ to the form $\exp (i \theta(t)), \exp (2 i \theta(t))$, resp., for $\theta(t)$ real-valued, either by appealing to Loewner's theory of slit mappings [5] or, even better, to the Carathéodory representation of $\hat{p}_{1}(t)$ and $\hat{p}_{2}(t)$ [1], [7]. Then (14) is equivalent to the inequality $-2[1+\cos \theta(t)]^{2} \leqq 0$ a.e., and the monotonicity of the real part of the third coefficient of $G$ is thereby proved.

This gives us Loewner's inequality $\operatorname{Re} \hat{a}_{3} \leqq 3$ when $\operatorname{Re} \hat{a}_{2} \geqq 0$, and at the same time shows that equality holds only when $\hat{f}$ is the Koebe function $z /(1-z)^{2}$. (If one inspects the real part of the second coefficient of $G$, it also is seen to be monotone decreasing, so the same Koebe function is extremal there, too.)

By a variant of the foregoing procedure one can prove a number of other inequalities, among which is Jenkins' inequality

$$
\operatorname{Re}\left[e^{2 i \phi}\left(a_{3}-a_{2}^{2}\right)-\lambda e^{i \phi} a_{2}\right] \leqq 1+3 \lambda^{2} / 8+\left(\lambda^{2} \log 4 / \lambda\right) / 4
$$

for $\phi$ real and $0<\lambda \leqq 4[8]$.

\section{REFERENCES}

1. G. S. Goodman, Univalent functions and optimal control, Thesis, Stanford University, California, 1968.

2. Chr. Pommerenke, Über die Subordination analytischer Funktionen, J. Reine Angew. Math. 218 (1965), 159-173.

3. C. Carathéodory, Vorlesungen über reelle Funktionen, Teubner, Leipzig, 1918.

4. G. S. Goodman, $A$ variational theory for Loewner's equation (to appear).

5. C. Loewner (K. Löwner), Untersuchungen über schlichte konforme Abbildungen des Einheitskreises, Math. Ann. 89 (1923), 103-121.

6. M. M. Schiffer, Sur l'equation différentielle de M. Löwener, C. R. Acad. Sci. Paris 221 (1945), 369-371.

7. C. Carathéodory, Über den Variabilitätsbereich der Fourier'schen Konstanten von positiven harmonischen Funktionen, Rend. Circ. Mat. Palermo 38 (1914), 193-217.

8. J. A. Jenkins, "On certain coefficients of univalent functions" in Analytic functions, Princeton Univ. Press, Princeton, N. J., 1960, pp. 159-193.

aarhus University, Aarhus, Demmark 